\title{
2
}

\section{Applications in Mechanics and Sensors}

\author{
Angel Rubio
}

\subsection{Introduction}

Since the discovery of carbon nanotubes (CNTs) by Sumio Iijima ${ }^{33}$ we witness an explosive development of the nanotube science and technology. Considering the rapid progress made in the fabrication, manipulation, characterization and modeling of nanostructures based on nanotubes, it is reasonable to expect that CNTs will pervade in the following years key application areas such as energy, materials, devices, and several others. There is a great variety of applications for which nanotubes represent a disruptive potential, ranging from energy storage, composites, nanoelectronics and other solid-state devices, to sensors and actuators (see for example the reviews by Baughman et al. ${ }^{5}$; Loiseau et al. $^{42}$ ).

Several structural varieties of nanotubes have been identified and classified based on criteria such as helicity (also known as chirality), number of walls, inclusion of pentagons-heptagons, etc. The simplest form is the single-walled carbon nanotube (SWNT) which resembles a rolled honeycomb graphite layer into a mono-atomic-thick cylinder. Several concentrically embedded SWNTs form a multi-walled carbon nanotube (MWNT). Other nanotube varieties include nanotube bundles or ropes, inter-tube junctions, nanotori, coiled nanotubes, etc. Due to their relative simplicity and atomically precise morphology, single-walled carbon nanotubes offer the opportunity of assessing the validity of different macro- and microscopic models.

The properties of carbon nanotubes can be grouped into three categories: structural, mechanical and electronic. From the structural point of view, in most situations, CNTs can be considered one-dimensional (1D) objects, with typical diameters $(\mathrm{dt})$ in the $\mathrm{nm}$-range and lengths $(\mathrm{L})$ reaching several micrometers. This one-dimensionality of tubes impacts on and is visible mostly through the mechanical and electronic properties. However, the structure of nanotubes can be exploited in itself such as for instance by field emitters or gas break-down sensors, which are based on the "sharpness" of CNTs giving rise to huge local electric fields. 


\section{$20 \mid 2$ Applications in Mechanics and Sensors}

While the prediction of electronic properties of carbon nanotubes required relatively subtle theoretical analysis, their unique mechanical behavior could be intuitively anticipated based on several features: strength of carbon bonds, their uniform arrangement within the graphitic sheet, and the seamless folding of this network into a tubule. The mechanical properties class is encompassing the elastic, thermal, vibrational or any other properties related to the motion of the tube's atoms. In nanotubes, carbon is $\mathrm{sp}^{2}$-hybridized resulting in strong $\sigma$-bonds weakly reinforced by $\pi$-bonds. Considering the hybridization, it is natural to assume a certain overlap between nanotube and graphite (graphene) elastic properties, such as Young's modulus, bending, tensile and torsional stiffness, and yield strength. SWNTs have tensile moduli close to 1TPa (stiff as diamond) and strengths $\approx 50 \mathrm{GPa}$ (corresponding to $5-10 \%$ maximal strain), which earned them the title of ultimate fibers. Despite their stiffness, CNTs retain a high bending flexibility due to their high aspect ratios $(\mathrm{L} / \mathrm{dt})$. With some exceptions, the thermal and vibrational properties of nanotubes also show similarities with graphite. Since the in-plane thermal conductivity of pyrolytic graphite is very high it is expected that the on-axis thermal conductivity of defect-free tubes would be even higher. At low temperatures the phonon mean free path is controlled mainly by boundary scattering and the coherence length $(\approx \mu \mathrm{m})$ is larger in tubules than in high oriented pyrolytic graphite $(<0.1 \mu \mathrm{m})$.

The class of electronic properties practically encompasses all remaining properties. Therefore it contains transport and electric properties (classical-, spindependent- and super-conductivity, dielectric permittivity), optical properties (absorbtion, scattering, luminescence), magnetic properties (susceptibility, Zeeman splitting, Aharonov-Bohm effect), chemical properties (covalent and non-covalent binding), as well as hybrid properties and correlated manybody effects (thermopower, piezoresistivity, piezoelectricity, Coulomb blockade, Kondo effect, Tomonaga-Luttinger liquid behavior). The electronic properties of nanotubes are strongly modulated by small structural variations, in particular, their metallic or semiconducting character is determined by the diameter and helicity (chirality) of the carbon atoms in the tube. A recent review of electronic and transport properties of carbon nanotubes can be found in Ref. 12 and on the fundamentals and applications of nanotubes in the books by Loiseau et al. ${ }^{42}$; Ebbesen ${ }^{24}$ and Dresselhaus et al..$^{22}$ (and references therein). 


\subsubsection{Some Applications: Carbon Nanotube-Based Sensors}

Continuously expanding, the field of CNT-based sensors includes an already impressive list of demonstrators, encompassing (bio)chemical, strain, stress, pressure, mass, flow, thermal, and optical sensors. The underlying mechanism of sensors involves the modulation of a CNT's electronic properties.

In Refs. 38 and 18 it is proposed to use CNTs as sensitive materials for chemical sensors. In Ref. 38 the electrical conductance of a nanotube was found to increase when the tube was exposed to $\mathrm{NO}_{2}$, and to decrease when exposed to $\mathrm{NH}_{3}$. Electron charge transfer (doping) was proposed as the mechanism dictating the change in conductivity (DG) by shifting the Fermi level of the channel. Collins et al. ${ }^{18}$ have shown that exposure to air or oxygen dramatically influences the nanotubes' electrical resistance, thermoelectric power, and local density of states, as determined by transport measurements and scanning tunneling spectroscopy. These electronic parameters can be reversibly "tuned" by surprisingly small concentrations of adsorbed gases, and semiconducting nanotubes can apparently be converted into metallic tubes through such exposure. Since these initial two reports, the list of chemFET-like gas and biological sensors has increased considerably. Innovations have also been brought, such as for example nanotube functionalization which has yielded highly sensitive and selective sensors. ${ }^{50,60}$ Also, recently Goldsmith et al. ${ }^{27}$ have demonstrated that it is possible to control the functionalization density by monitoring in real-time the conductance of a nanotube in an electrochemical setup.

Ghosh et al. ${ }^{26}$ report flow sensors, in which the conductance of carbon nanotubes, disposed parallel to the flow lines, is clearly changed at different flow rates. The suggested mechanism for this nonlinear effect is forcing of the electrons in the nanotubes by the fluctuating Coulombic field of the liquid, or, briefly, Coulomb drag. Another CNT flow-meter demonstrated recently by Bourlon et al. ${ }^{8}$ operates differently from the previous device. It is also worthy to mention that reading-out the modulation of the electronic properties in chemical sensor needs not be confined to electrical measurements. A promising approach is using light to probe either the photoabsorption or the fluorescence spectra. ${ }^{11,29}$ Cao et al. ${ }^{11}$ have studied band-gap photo-absorption in SWNTs, previously exposed to air, in different organic solvents. 


\section{$22 \mid 2$ Applications in Mechanics and Sensors}

An ionization CNT sensor has been demonstrated by Modi et al. ${ }^{47}$ based on the ionization fingerprint which is characteristic to each analyte gas. This device simply exploits the geometry of the nanotubes, since their sharp tips generate very high electric fields at relatively low voltages, lowering gas breakdown voltages. Another category of sensor demonstrators that make use of CNTs as electrodes are the amperometric biosensors utilizing nanotubes as electrode material in electrochemical setups. This type of sensors are exhaustively reviewed in Wang, ${ }^{67}$ Still other CNT sensing devices could be mentioned here, such as low temperature quantum electrometers ${ }^{52}$ and superconducting quantum interference device (SQUID) magnetometers. ${ }^{17}$

\subsection{Nanotube Nanomechanics}

Carbon as well as composite BN nanotubes demonstrate very high stiffness to an axial load or a bending of small amplitude, which translates in the recordhigh efficient linear-elastic moduli. At larger strains, the nanotubes (especially, the single-walled type) are prone to buckling, kink forming and collapse, due to the hollow shell-like structure. These abrupt changes (bifurcations) manifest themselves as singularities in the non-linear stress-strain curve, but are reversible and involve no bond-breaking or atomic rearrangements. This resilience corresponds, quantitatively, to a very small sub-angstrom efficient thickness of the constituent graphitic shells. Irreversible yield of nanotubes begins at extremely high deformation (from several to dozens percent of in-plane strain, depending on the strain rate) and high temperature. ${ }^{a}$ The atomic relaxation begins with the edge dislocation dipole nucleation, which (in case of carbon) involves a diatomic interchange, i. e. a $90^{\circ}$ bond rotation. ${ }^{\text {b }}$ A sequence of similar diatomic steps ultimately leads to failure of the nanotube filament. The failure threshold (yield strength) turns out to depend explicitly on nanotube helicity, which thus demonstrates again the profound role of symmetry for the physical properties, either electrical conductivity or mechanical strength. Finally, the manifestation of mechanical strength in the multi-walled or bundled nanotubes (ropes) is obscured by the poor load transfer from the exterior to the core of

\footnotetext{
aNote that the temperature is an important parameter in the strength of a material since the motion of dislocations is thermally activated. Like all covalent materials, nanotubes are brittle at low temperatures. The flexibility of the nanotubes at room temperature is due to their high strength and the unique ability of the hexagonal network to distort in order to release the applied stress.

${ }^{b}$ This bond-rotation defects are more unlikely in boron nitride due to the larger energy needed to form B-B or N-N bonds. This could increase the yield strength of the nanotube as compared to carbon.
} 
such larger structure. This will lead to lower apparent strength and even lower linear moduli, as they become limited by the weak lateral interaction between the tubules rather than by their intrinsic carbon bond network. The ultimate strength of nanotubes and their ensembles is an issue that requires the modeling of inherently mesoscopic phenomena, such as plasticity and fracture, on a microscopic, atomistic level, and constitutes a challenge from the theoretical as well as experimental points of view. A recent very nice overview assessing carbon nanotube strength is given in Dumitrica et al..$^{23}$

The similarities among graphite and other $\mathrm{sp}^{2}$-like bonded materials as hexagonal boron nitride and boron-carbon-nitrogen compounds, lead us to theoretical proposition that $\mathrm{B}_{x} \mathrm{C}_{y} \mathrm{~N}_{z}$ nanotubes would be stable. ${ }^{43,44,53,54}$ Specific synthesis of these nanotubes was achieved afterwards: boronnitride ${ }^{15,41}$ and $\mathrm{BC}_{2} \mathrm{~N}$ and $\mathrm{BC}_{3}{ }^{61,68}$ as well as other inorganic tubular forms of $\mathrm{WS}_{2}$ and $\mathrm{MoS}_{2}{ }^{62}$ The predicted properties of these tubules are quite different from those of carbon with numerous possible technological applications in the fields of catalysis, lubrication, electronic and photonic devices. ${ }^{43,44, c}$

Calculations of the stiffness of SWNTs demonstrated that the Young modulus shows a small dependence on the tube diameter and chirality for the experimental range of nanotube diameters (between 1.3 and $1.4 \mathrm{~nm}$ ). It is predicted that carbon nanotubes have the highest Young's modulus of all the different types of composite tubes considered $\left(\mathrm{BN}, \mathrm{BC}_{3}, \mathrm{BC}_{2} \mathrm{~N}, \mathrm{C}_{3} \mathrm{~N}_{4}, \mathrm{CN}\right){ }^{31}$ Those results for the $\mathrm{C}$ and $\mathrm{BN}$ nanotubes are reproduced in the left panel of Fig. 1. Furthermore, the Young modulus approaches, from below, the graphitic limit for diameters of the order of $1.2 \mathrm{~nm}$. The computed value of $\mathrm{C}$ for the wider carbon nanotubes of $0.43 \mathrm{TPa} \mathrm{nm}$, that corresponds to $1.26 \mathrm{TPa}$ modulus in our convention, is in excellent agreement with the experimental value for SWNT's of $1.25 \mathrm{TPa}^{66}$ It is also in rather good agreement with the value of 1.28 TPa reported for multi-wall nanotubes (MWNT) ${ }^{67}$ Although this result is for MWNT the similarity between SWNT is not surprising as the intra-wall $\mathrm{C}-\mathrm{C}$ bonds mainly determine the Young's modulus. From these results we can

${ }^{\mathrm{c}}$ The electronic properties of $\mathrm{BN}$ nanotubes are quite different to carbon, namely: all are stable wide band-gap semiconductors independent of helicity and diameter of the nanotube and of whether the nanotube is single- or multi-walled. On the other hand, single-wall $\mathrm{BC}_{3}$ tubules are found to be semiconductor with a small gap of $\sim 0.5 \mathrm{eV}$ that would disappear in the multilayer form. ${ }^{43,44}$ The inter-wall interaction for concentric tubules makes the conduction band overlap with the valence band maximum ( $\sigma$-bands). Therefore, a concentric needle of $\mathrm{BC}_{3}$ tubules will have $\sigma$ conductivity and opens the question of how many walls are necessary to give rise to conductivity in concentric $\mathrm{BC}_{3}$ tubules, that is to look for a semiconductor-metal transition as a function of the number of concentric shells. 

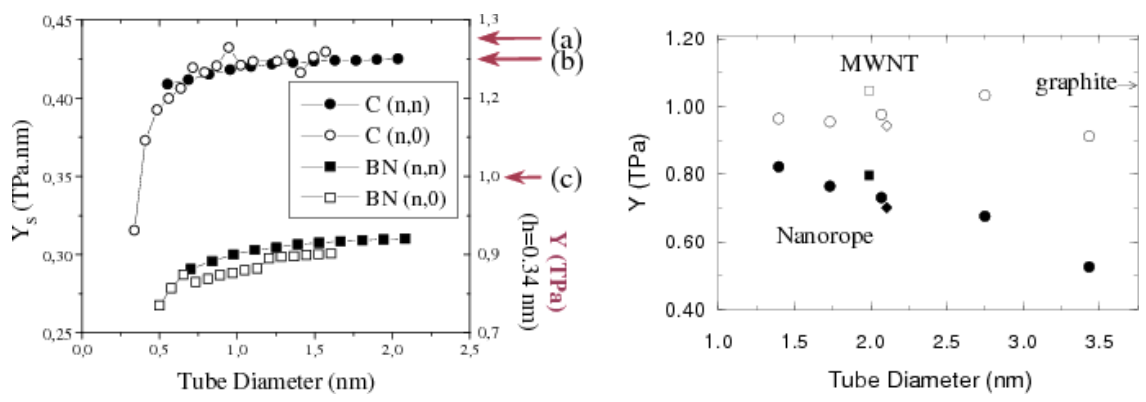

Fig. 1. Left: Young modulus for armchair and zig-zag carbon and $B N$ nanotubes. The values are given in the proper unit of TPa nm for SWNTs (left axis), and converted to TPa (right axis) by taking a value for the graphene thickness of $0.34 \mathrm{~nm}$. The experimental values for carbon nanotubes are reported on the right-hand-side: (a) $1.28 \mathrm{TPa}^{69}$; (b) $1.25 \mathrm{TPa}^{39}$; (c) $1 \mathrm{TPa}$ for MWNTs. ${ }^{48}$ Right: Young modulus versus tube diameter in different arrangements. Open symbols correspond to the multi-wall geometry (10-layer tube with an interlayer distance of $0.34 \mathrm{~nm}$ ), and solid symbols for the single-wall-nanotube crystalline-rope configuration. In the MWNT geometry the value of the Young modulus does not depend on the specific number of layers. The experimental value of the $c_{11}=1.06 \mathrm{TPa}$ elastic constant of graphite is also shown..$^{58}$

estimate the Young modulus considering two different geometries of practical relevance:

(1) multi-wall like geometry, in which the normal area is calculated using the wall-wall distance as the one in MWNTs, which is very approximately equal to the one of graphite,

(2) nanorope or bundle configuration of SWNTs, where the tubes would be arranged forming an hexagonal closed packed lattice, with a lattice constant of $(2 R+3.4)$, being $R$ the tube radius.

The results for these two cases are presented in the right panel of Fig. 1. The MWNT geometry give a value that is very close to the graphitic one, however the rope geometry shows a decrease of the Young modulus with increasing the tube radius due to the quadratic increase of the effective area in this configuration, while the number of atoms increases only linearly with the tube diameter. The computed values for the SWNT ropes experimentally observed are, however, still very high $(0.5 \mathrm{TPa})$, even comparing with other known carbon fibers. This value is in quite good agreement with AFM experiments on anchored SWNTs ropes ${ }^{57}(\mathrm{Y} \sim 0.6 \mathrm{TPa})$ and for stress-strain puller measurements of the Young modulus for aligned nanotube ropes of MWNTs $(\mathrm{Y} \sim 0.45 \pm 0.23 \mathrm{TPa}) .{ }^{49}$ We should remark that this apparent "low" $\mathrm{Y}$ is not due to the presence of defects as arguments in Pan, ${ }^{49}$ and can be understood 
in simple geometrical terms by the particular "empty" cross-sectional area is responsible for the apparent lower modulus.

For composite nanotubes, the results of Chopra ${ }^{16}$ for the Young modulus of BN MWNT's of $1.22 \mathrm{TPa}$, which is somewhat larger than the result obtained for these tubes in the TB calculations $(\sim 0.9 \mathrm{TPa}),{ }^{30}$ but nevertheless the agreement is close. ${ }^{\mathrm{d}}$ We indicate that $\mathrm{BN}$ and $\mathrm{BC}_{3}$ tubes have similar values of the Young modulus, ${ }^{\mathrm{e}}$ although the latter have slightly larger values. In those studies $\mathrm{C}_{3} \mathrm{~N}_{4}$ nanotubes are shown to be much softer than any other type of tube, the reason being that for a given amount of tube surface, there is a smaller density of chemical bonds. ${ }^{31}$

The Poisson ratio is given by the variation of the radius of the SWNT resulting from longitudinal deformations along the tube axis. In all cases the computed Poisson ratio is positive: an elongation of the tube reduces its diameter. The ab initio values are 0.14 (from 0.12 to 0.16 ) for the armchair $(\mathrm{n}, \mathrm{n}$ ) tubes, and a little larger for other chiralities: 0.19 for $(10,0)$ and 0.18 for $(8,4)$. The uncertainty of the obtained values is of the order of $10 \%$. In summary, the $a b$ initio calculations indicate that the Poisson ratio retains graphitic values except for a possible slight reduction for small radii. It shows chirality dependence: $(n, n)$ tubes display smaller values than $(10,0)$ and $(8,4)$. Similar differences are found between the $a b$ initio and TB calculations for $\mathrm{BN}$ tubes, namely for the $(6,6)$ tube the ab initio value for the Poisson ratio is 0.23 whereas the $\mathrm{TB}$ one is $0.30 .{ }^{30}$

Large amplitude deformations, beyond the Hookean behavior, reveal nonlinear properties of nanotubes, unusual for other molecules or for the graphite fibers. Both theory-simulations and experimental evidence suggest the ability of nanotubes to significantly change their shape, accommodating to external forces without irreversible atomic rearrangements. They develop kinks or the ripples (multi-walled tubes) in compression and bending, flatten into deflated "ribbons" under torsion, and still can reversibly restore original shape. This resilience is unexpected for a graphite-like material. It must be attributed to the small dimension of the tubules, which leaves no room for the stressconcentrators - micro-cracks or dislocation piles, making a macroscopic material prone to failure. Furthermore, MWNT can be bent repeatedly through large angles using the tip of an atomic force microscope without undergoing catastrophic failure. ${ }^{25}$ The observed response at very high strain deformation

\footnotetext{
${ }^{\mathrm{d}}$ The smaller Young modulus for $\mathrm{BN}$ compared to $\mathrm{C}$-nanotubes is directly related to the difference in the experimental $\mathrm{c}_{11}$ elastic constants that are $0.75 \mathrm{TPa}$ and $1.06 \mathrm{TPa}$ for hexagonal-BN and graphite, respectively.

The calculated average Young modulus is $0.9 \mathrm{TPa}$ and $0.92 \mathrm{Tpa}$ for $\mathrm{BN}$ and $\mathrm{BC}_{3}$, respectively.
} 
indicates that nanotubes are remarkably flexible and resilient. The main outcome of mechanical studies of carbon nanotubes is that both thin- and thickwalled carbon nanotubes exhibit compressive strengths about two orders of magnitude higher than any other known fiber. ${ }^{f}$

\subsubsection{Experimental Evidence of Nanotube Resilience}

Collapsed forms of the nanotube ("nanoribbons") have been observed in experiment (Fig. 2d) and their stability can be explained by the competition between the van der Waals attraction and elastic energy. The basic physics can be understood by noticing that the elastic curvature energy per unit length is proportional to $1 / \mathrm{R}$ ( $\mathrm{R}$, radii of the tube); however, for a fully collapsed singlewall tubule with the opposite tubule walls at the typical van der Waals contact distance c, the energy per unit length would be composed of a higher curvature energy due to the edges which is independent of the initial tubule diameter,
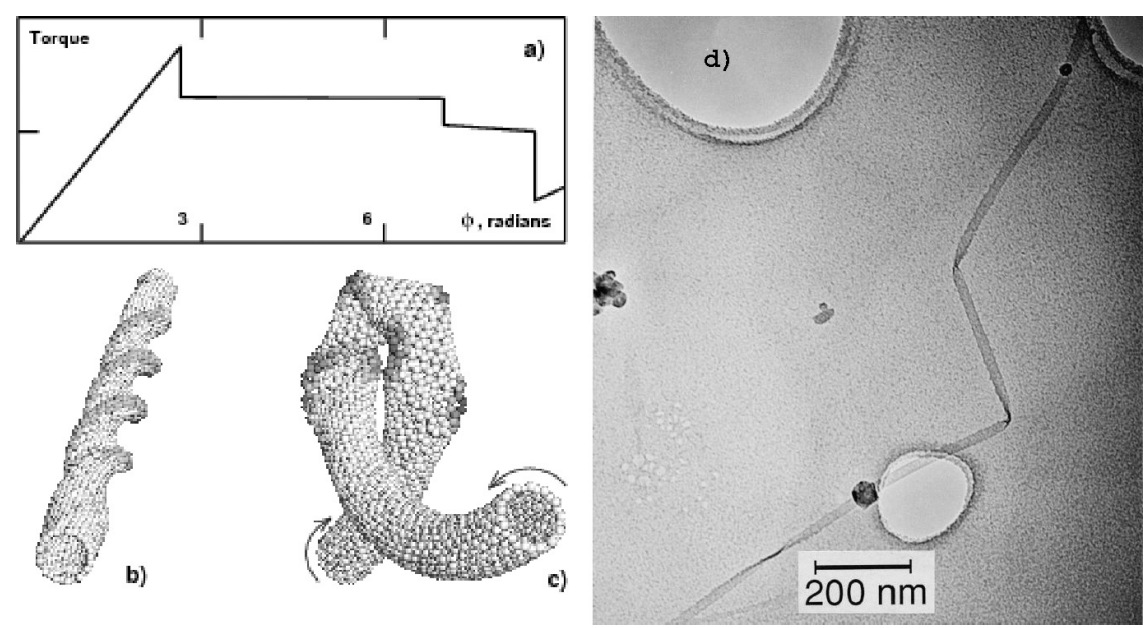

Fig. 2. Simulation of torsion and collapse. ${ }^{71}$ The strain energy of a $25 \mathrm{~nm}$ long $(13,0)$ tube as a function of torsion angle $\phi(a)$. At first bifurcation the cylinder flattens into a straight spiral (b) and then the entire helix buckles sideways, and coils in a forced tertiary structure (c). Collapsed tube (d) as observed in experiment. ${ }^{14,15}$

\footnotetext{
${ }^{f}$ Calculations of the elastic properties of $\mathrm{C}$-nanotubes confirm that they are extremely rigid in the axial direction (high-tensile strength) and more readily distort in the perpendicular direction (radial deformations), due to their high aspect ratio. The detailed studies, stimulated first by experimental reports of visible kinks, lead one to conclude that, in spite of their molecular size, nanotubes obey very well the laws of continuum shell theory. $3,40,65$
} 
and a negative van der Waals contribution, $\varepsilon_{\mathrm{vdW}} 0.03-0.04 \mathrm{eV} / \mathrm{atom}$, that is $\propto \mathrm{R}$ per unit length. Collapse occurs when the latter term prevails above a certain critical tube radii $\mathrm{R}_{\mathrm{c}}$ that depends on the number $\mathrm{N}$ of shells of the nanotube, $\mathrm{R}_{\mathrm{c}}(\mathrm{N}=1) \sim 8 \mathrm{c}$ and $\mathrm{R}_{\mathrm{c}}(\mathrm{N}=8) \sim 19 \mathrm{c},{ }^{14}$ and the thickness of the collapsed strip-ribbon is $(2 \mathrm{~N}-1) \mathrm{c}$. Any additional torsional strain imposed on a tube in experimental environment also favors flattening ${ }^{70-73}$ and facilitates the collapse, (Figs. 2b-c).

The bending seems fully reversible up to very large bending angles despite the occurrence of kinks and highly strained tubule regions in simulations, that are in excellent morphological agreement with the experimental images. ${ }^{33}$ Similar bent-buckled shapes have been reported by several groups ${ }^{20,33,55}$ (Fig. 2). This apparent flexibility stems from the ability of the $\mathrm{sp}^{2}$ network to rehybridize when deformed out of plane, the degree of $\mathrm{sp}^{2}-\mathrm{sp}^{3}$ rehybridization being proportional to the local curvature. ${ }^{28}$

The accumulated evidence thus suggests that the strength of the carboncarbon bond does not guarantee resistance to radial, normal to the graphene plane deformations. In fact, the graphitic sheets of the nanotubes, or of a plane graphite $^{32}$ though difficult to stretch are easy to bend and to deform. A crystalarray $^{64}$ or a pair ${ }^{56}$ of parallel nanotubes flatten at the lines of contact between them so as to maximize the attractive van der Waals intertube interaction. Computer simulations have provided a compelling evidence of the mechanisms discussed above. By carefully tuning the tension in the tubule and gradually elevating its temperature, with extensive periods of MD annealing, the first stages of the mechanical yield of CNT have been observed. ${ }^{22}$ In simulation of tensile load the novel patterns in plasticity and breakage, just described above, has clearly emerged.

\subsection{Applications of Mechanical Response}

The understanding of the mechanical response of nanotubes to external forces is of relevance for the application of nanotubes as a composite material reinforcement as well as in electronic devices, where the deformation of the tubes induced by the substrate alter locally the electronic properties of the nanotube. A broad discussion of potential applications of the nanotube can be found in existing reviews, ${ }^{1,6,24,73}$ also outlining the challenges of implementation. There are two already actual applications, where the carbon nanotube can commercially compete with other materials units, and the mechanics of carbon nanotube plays either central or an important secondary role in both cases. 
Different to traditional graphitic fibers, nanotubes combine high flexibility and high strength with high stiffness. These properties open the way for a new generation of high performance light-weight polymer composites composites useful for structural reinforcement. This has triggered a lot of research looking at the ability of nanotubes to stiffen and strengthen a polymer. ${ }^{1,59}$ The reinforcement will depend on how load is transferred to the nanotube aggregates such as single-walled nanotube bundles. If the adhesion between the matrix and the nanotubes is not strong enough to sustain high loads, the benefit of the high tensile strength of carbon nanotubes are lost. Load transfer in carbon nanotube epoxy composites was studied in both tension and compression. ${ }^{59}$ Under tension it seems that only the outermost nanotube is loaded due to weak interlayer bonding. This could be related to the absence of registry between graphene layers that makes adhesion and friction decresed. ${ }^{25}$ The excepcional mechanical properties of nanotubes will be reflected in composites once a good load transfer between the matrices and outer surface of the nanotubes is obtained.

Nanotubes are ideal proximal tips because the do not plastically deform during tip crashes on the surfaces as conventional tips often do. Instead they elastically deform, buckle and slip. The recovering mechanism has to be taken into account when analyzing the data from AFM measurements using the nanotubes as tips. The enhanced capability of a scanning force microscope using carbon nanotube has been already demonstrated. ${ }^{19}$ Nanotube tips have also been used as pencils for writing 10 -nm-width structures on silicon substrates. The robustness of the nanotube tips permits a writing rate $0.5 \mathrm{~mm} / \mathrm{sec}$, five times faster than was possible with older AFM tips. The way the nanotube writes is for an electric field, emanating from the nanotube, to remove hydrogen atoms from a layer of hydrogen atop a silicon base. The exposed silicon surface oxidizes; thus the "writing" consists of narrow $\mathrm{SiO}_{2}$ track.

The development of new tools to manipulate and analyze the nanoworld relies in having traditional macrocopic techniques down to the nanoscale. Just recently it has been probed that the mechanical robustness and electrical conductivity of nanotubes can be used to build electromechanical-tweezers. ${ }^{37}$ The nanotubes are attached to a metal electrode. This tweezers not only allow to grab and manipulate mesoscopic silicon-carbide dots and gallium-arsenide wires but also can be used to probe electronic properties across the structure (transport). Many other applications can be envisioned, for example, the manipulation of biological structures.

A full-sealed field emission display has been recently reported by the group at Samsung Corporation ${ }^{13}$ who used a relatively disordered array of 
SWNT embedded in a composite substrate, in combination with closelyplaced $(200 \mathrm{~mm})$ phosphorous layer. At the SWNT density of only $1-3 \mathrm{~mm}^{2}$, the current density is stable for hours and high enough to induce bright light. In this important example, mechanical robustness of the carbon network in the tips prevents them from deterioration.

Mechanical behavior is often coupled with other physico-chemical phenomena, which of course broadens the import of mechanical properties, for example Elasto-electronics: Coupling with electrical properties can manifest itself both ways, where either deformation affects the charge distribution or transport, or an additional charge can cause visible deformation, thus making an actuator. Change in the bandgap of nanotubes with strain and torsion has been discussed. ${ }^{36, g}$ This variation could have some implications for nanoscale electro-mechanical devices. ${ }^{35}$

Furthermore, bending of a carbon nanotube introduces an increased mixing of $\sigma$ and $\pi$ states that leads to an enhanced density of states at the Fermi energy region and to a charge polarization of the carbon-carbon bond in the deformed region. ${ }^{51}$ The transport properties of bent tubes depend on the chirality of the tube, indeed, armchair tubes keep their metallic character irrespective of the deformation but this is not the case for chiral metallic tubes where localelectronic barriers arise from the bending of the tube. ${ }^{9,10}$ This calculations are in agreement with recent experimental transport data of individual carbon nanotubes supported on a series of electrodes. ${ }^{\text {? }}$

Mechanical stretching has been proposed as a method for inducing chiral conductivity in these carbon nanotubes when doped. ${ }^{45}$ The essence of chiral conduction is symmetry breaking on tubule walls upon mechanical stretching. Calculations of the transport properties of mechanically stretched dopedcarbon nanotubes show that stretching induces chirality on the tubule current, being the most efficient geometry for the induced chirality an arm-chair like atomic arrangement. If a nanocoil is subjected to an external frequencydependent electric field the effective inductance varies with respect to ACfrequency different to the inductances of ordinary coils. This induced current chirality is most likely for geometrically chiral nanotube having helical pitches close to those of armchair tubules. By solving Maxwell's equations for chiral conducting nanotubes (nanocoils) it is found that the self-inductance and the resistivity of nanocoils should depend on the frequency of the alternating

\footnotetext{
${ }^{g}$ The coupling between the conduction electrons and long wavelength twistons, i.e., torsional shape vibrations is relevant for understanding the transport properties at low temperatures, namely, the relevance for the peculiar linear dependence of the electrical resistivity with temperature in the metallic armchair tubes.
} 
$30 \mid 2$ Applications in Mechanics and Sensors

current even when the capacitance of the nanocoils is not taken into account. ${ }^{46}$ This is in contrast to elementary treatment of ordinary coils. This fact is useful to distinguish nanocoils by electrical measurements. ${ }^{\mathrm{h}}$

An interesting manifestation of electro-mechanical coupling in nanotubes has recently emerged as carbon nanotube actuators. ${ }^{4}$ By changing the applied voltage and therefore injecting the electrons or holes one causes either an expansion or contraction of carbon nanotube, or a graphene sheet. A separate contribution of a purely quantum-mechanical change in band structure and in orbital occupancy and the role of electrochemical double-layer are not completely understood, the experimental evidence is quite convincing. Nanotube sheets adhered to the opposite sides of insulating film make a cantilever sensitive to the voltage applied between the sheets: a small elongation on one side and a contraction on the other results in significant bending of the bilayer cantilever. The limit of the gravimetric work capacity, $1 / 2 \mathbf{Y}_{\varepsilon}^{2} / \rho$, is expected to be much higher than for other materials, like ferroelectrics, due to great values of modulus $\mathrm{Y}=1 \mathrm{TPa}$, strain range $\varepsilon=1 \%$, and the low density $\rho=1.3 \mathrm{~g} / \mathrm{cm}^{3}$ for carbon nanotube bundles.

\section{Acknowledgements}

We are grateful to S. Roche, C. Roman, V. Crespi, M. Cohen, S. G. Louie, P. M. Ajayan and acknowledge funding from the European Community through NoE Nanoquanta (NMP4-CT-2004-500198), SANES (NMP4-CT2006-017310), DNA-NANODEVICES (IST-2006-029192) and NANOERAChemistry projects, UPV/EHU (SGIker Arina) and Basque Country University (SGIKer ARINA) and "Grupos Consolidados UPV/EHU del Gobierno Vasco" (IT-319-07) and e-I3 ETSF project (INFRA-2007-1.2.2: Grant Agreement Number 211956). We acknowledges the computer resources, technical expertise and assistance provided by the Barcelona Supercomputing Center-Centro Nacional de Supercomputación.

\section{Bibliography}

1. Ajayan, P. M. and Ebbesen, T. W. (1997) Reports on Progress in Physics, 60, 1025.

2. Ajayan, P. M., Schadler, L. S., Giannaris, C. and Rubio, A. (2000) Adv. Mat., 12, 750.

\footnotetext{
${ }^{\mathrm{h}}$ The basic physics behind this phenomenon is that in a nanocoil the chiral angle of the current is frequency dependent whereas in a classical coil this is fixed by the pitch of the winding of the wires in forming the coil.
} 
3. Allen, H. G. and Bulson, P. S. (1980) Background to Buckling, McGraw-Hill, London, p. 582.

4. Baugman, R. H., et al. (1999) Science, 284, 1340.

5. Baugman, R. H., et al. (2002) Science, 297, 787.

6. Bernholc, J., Roland, C. and Yakobson, B. I. (1997) Current Opinion in Solid State $\mathcal{E}$ Materials Science, 2, 706-715.

7. Bezryadin, A., Verschueren, A. R. M., Tans, S. J. and Dekker, C. (1999) Phys. Rev. Lett., 80, 4036.

8. Bourlon, B., Wong, J., Mikó, C., Forró, L. and Bockrath, M. (2007) Nature Nanotech., $2,104$.

9. Buongiorno-Nardelli, M., Yakobson, B. I. and Bernhold, J. (1998) Phys. Rev. B, 57, 4277.

10. Buongiorno-Nardelli, M., Yakobson, B. I. and Bernhold, J. (1998) Phys. Rev. Lett., 81, 4656.

11. Cao, A., Talapatra, S., Choi, Y. Y., Vajtai, R., Ajayan, P. M., Filin, A., Persans, P. and Rubio, A. (2005) Advanced Materials, 17, 147.

12. Charlier, J.-C., Blasé, X. and Roche, S. (2007) Rev. Mod. Phys., 79, 677.

13. Choi, W. B., et al. (1999) Appl. Phys. Lett., 75, 3129.

14. Chopra, N. G., Benedict, L. X., Crespi, V. H., Cohen, M. L., Louie, S. G. and Zett, A. (1995a) Nature, 377, 135.

15. Chopra, N. G., Luyken, R. J., Cherrey, K., Crespi, V. H., Cohen, M. L., Louie, S. G. and Zettl, A. (1995b) Science, 269, 966.

16. Chopra, N. G. and Zettl, A. (1998) Solid State Comm., 105, 297.

17. Cleuziou, J.-P., Wernsdorfer, W., Bouchiat, V., Ondarçuhu, T. and Monthioux, M. (2006) Natl. Nanotech., 1, 53.

18. Collins, P. G., Bradley, K., Ishigami, M. and Zettl, A. (2000) Science, 287, 1801.

19. Dai, H., Hafner, J. H., Rinzler, A. G., Colbert, D. T. and Smalley, R. E. (1996) Nature, $384,147$.

20. Despres, J. F., Daguerre, E. and Lafdi, K. (1995) Carbon, 33, 87.

21. Dresselhaus, M. S., Dresselhaus, G. and Eklund, P. C. (1996) Science of Fullerenes and Carbon Nanotubes, Academic Press, San Diego, p. 965.

22. Dresselhaus, M. S., Dresselhaus, G., Sugihara, K., Spain, I. L. and Goldberg, H. A. (1998) Graphite Fibers and Filaments, Springer-Verlag, Heidelberg, p. 382.

23. Dumitrica, T., Hua, M. and Yakobson, B. (2006) Proc. Natl. Acad. Sci., 103, 6105.

24. Ebbesen, T. W., ed., (1997) Carbon Nanotubes: Preparation and Properties, CRC Press, Tokyo, p. 296.

25. Falvo, M. R., Clary, G. J., Taylor, R. M., Chi, V., Brooks, F. P., Washburn, S. and Superfine, R. (1997) Nature, 389, 582.

26. Ghosh, S., Sood, A. K. and Kumar, N. (2003) Science, 299, 1042.

27. Goldsmith, B. R., et al. (2007) Science, 315, 77.

28. Haddon, R. C. (1993) Science, 261, 1545.

29. Heller, D. A., et al. (2006) Science, 311, 508. 
$32 \mid 2$ Applications in Mechanics and Sensors

30. Hernández, E., Goze, C., Bernier, P. and Rubio, A. (1998) Phys. Rev. Lett., 80, 4502.

31. Hernández, E., Goze, C., Bernier, P. and Rubio, A. (1999) Appl. Phys. A., 68, 287.

32. Hiura, H., Ebbesen, T. W., Fujita, J., Tanigaki, K. and Takada, T. (1994) Nature, 367, 148.

33. Iijima, S. (1991) Nature, 354, 56.

34. Iijima, S., Brabec, C., Maiti, A. and Bernholc, J. (1996) J. Chem. Phys., 104, 2089.

35. Joachim, C. and Gimzewski, J. K. (1997) Chem. Phys. Lett., 265, 353.

36. Kane, C. L. and Mele, E. J. (1997) Phys. Rev. Lett., 78, 1932.

37. Kim, P. and Lieber, C. M. (1999) Science, 286, 2149.

38. Kong, J., et al. (2000) Science, 287, 622.

39. Krishnan, A., Dujardin, E., Ebbesen, T. W., Yanilos, P. N. and Treacy, M. M. J. (1998) Phys. Rev. B, 58, 14013.

40. Landau, L. D. and Lifschitz, E. M. (1975) Theory of Elasticity, 3rd ed., Pergamon, Oxford.

41. Loiseau, A., Willaime, F, Demoncy, N., Hug, G. and Pascard, H. (1996) Phys. Rev. Lett., 76, 4737.

42. Loiseau, A., Launois, P., Pteit, P., Roche, S. and Salvetat, J.-P. (2006) Understanding Carbon Nanotubes, Lecture Notes in Physics, Springer Verlag, Vol. 677.

43. Miyamoto, Y., Rubio, A., Louie, S. G. and Cohen, M. L. (1994a). Phys. Rev. B, 50, 4976.

44. Miyamoto, Y., Rubio, A., Louie, S. G. and Cohen, M. L. (1994b) Phys. Rev. B, 50 18360.

45. Miyamoto, Y. (1996) Phys. Rev. B, 54, R11149.

46. Miyamoto, Y., Rubio, A., Louie, S. G. and Cohen, M. L. (1999) Phys. Rev. B, 60, 13885.

47. Modi, A., Koratkar, N., Lass, E., Wei, B. and Ajayan, P. M. (2003) Nature, 424, 171.

48. Muster, J., Burghard, M., Roth, S., Düsberg, G. S., Hernández, E. and Rubio, A. (1998) J. Vac. Sci. Technol., 16, 2796.

49. Pan, Z. W., Xie, S. S., Lu, L., Chang, B. H., Sun, L. F., Zhou, W. Y., Wang, G. and Zhang, D. L. (1999) Appl. Phys. Lett., 74, 3152.

50. Qi, P., et al. (2003) Nano Lett., 3, 347.

51. Roschefort, A., Avouris, P. and Salahub, D. R. (1999) Phys. Rev. B, 60, 13824.

52. Roschier, L., Tarkiainen, R., Ahlskog, M., Paalanen, M. and Hakonen, P. (2001) Appl. Phys. Lett., 78, 3295.

53. Rubio, A., Corkill, J. L. and Cohen, M. L. (1994) Phys. Rev. B, 49, 5081.

54. Rubio, A. (1997) Cond. Matt. News, 6, 6.

55. Ruoff, R. S. and Lorents, D. C. (1995) Bulletin of the APS, 40, 173.

56. Ruoff, R. S., Tersoff, J., Lorents, D. C., Subramoney, S. and Chan, B. (1993) Nature, $364,514$.

57. Salvetat, J. P., Briggs, G. A. D., Bonard, J. M., Bacsa, R. R., Kulik, A. J., Stöckli, T., Burnham, N. A. and Forro, L. (1999) Phys. Rev. Lett., 82, 944.

58. Sánchez-Portal, D., Artacho, E., Soler, J. M., Rubio, A. and Ordejón, P. (1999) Phys. Rev. B, 59, 12678. 
59. Schadler, L. S., Giannaris, S. C. and Ajayan, P. M. (1998) Appl. Phys. Lett.

60. Staii, C. and Johnson, A. T. (2005) Nano Lett., 5, 1774.

61. Stephan, O., Ajayan, P. M., Colliex, C., Redlich, P., Lambert, J. M., Bernier, P. and Lefin, P. (1994) Science, 266, 1683.

62. Tenne, R., Margulis, L., Genut, M. and Hodes, G. (1992) Nature, 360, 444.

63. Tenne, R. (1995) Adv. Mater., 7, 965; and references therein.

64. Tersoff, J. and Ruoff, R. S. (1994) Phys. Rev. Lett., 73, 676.

65. Timoshenko, S. P. and Gere, J. M. (1998) Theory of Elastic Stability, McGraw-Hill, New York, p. 541.

66. Treacy, M. M. J., Ebbesen, T. W. and Gibson, J. M. (1996) Nature, 381, 678.

67. Wang, J. (2005) Electroanalysis, 17, 7.

68. Weng-Sieh, Z., Cherrey, K., Chopra, N. G., Blase, X., Miyamoto, Y., Rubio, A., Cohen, M. L., Louie, S. G., Zettl, A. and Gronsky, R. (1995) Phys. Rev. B, 51, 11229.

69. Wong, E. W., Sheehan, P. E. and Lieber, C. M. (1997) Science, 277, 1971.

70. Yakobson, B. I., Brabec, C. J. and Bernholc, J. (1996a) Phys. Rev. Lett., 76, 2511.

71. Yakobson, B. I., Brabec, C. J. and Bernholc, J. (1996b) J. Computer-Aided Materials Design, 3, 173.

72. Yakobson, B. I., Campbell, M. P., Brabec, C. J. and Bernholc, J. (1997a) Computational Materials Science, 8, 341.

73. Yakobson, B. I. and Smalley, R. E. (1997b) American Scientist, 85, 324. 
